\title{
Activation of the AKT Pathway in the Ascending Aorta With Bicuspid Aortic Valve
}

\author{
Yuichiro Hirata, MD; Hiroki Aoki, MD, PhD; Takahiro Shojima, MD, PhD; \\ Kazuyoshi Takagi, MD, PhD; Tohru Takaseya, MD, PhD; Kohji Akasu, MD, PhD; \\ Satoru Tobinaga, MD, PhD; Yoshihiro Fukumoto, MD, PhD; Hiroyuki Tanaka, MD, PhD
}

\begin{abstract}
Background: Dilatation of the ascending aorta affects those patients with bicuspid aortic valve (BAV), even after valvular surgery, possibly due to tissue fragility. The goal of the study was the molecular characterization of aorta with BAV compared to that with normal tricuspid aortic valve (TAV).
\end{abstract}

\begin{abstract}
Methods and Results: The subjects were patients who underwent surgery for aortic valve stenosis in 2013 and 2014 . Nine patients with BAV and 13 with TAV were examined. There was no difference in the clinical characteristics or grade of aortic valve stenosis, but the diameters of the ascending aorta were significantly higher in the BAV group. The ascending aortic specimens were subjected to transcriptome analyses, which revealed the changes in receptor tyrosine kinase (RTK) pathway-related genes between TAV and BAV samples. Immunohistochemical study revealed higher staining of phosphorylated AKT (pAKT) in the media of the ascending aorta in the BAV group, regardless of the size of ascending aorta, whereas total AKT did not show such a difference. Immunofluorescence staining revealed the AKT activation was mainly in the medial vascular smooth muscle cells.
\end{abstract}

Conclusions: The results showed that the RTK-AKT pathway in the medial layer of the ascending aorta is activated in aortae with BAV. Activation of this pathway may be associated with fragility and dilatation of the ascending aorta with BAV.

Key Words: AKT; Bicuspid aortic valve; Transcriptome

B icuspid aortic valve (BAV) is a congenital cardiovascular malformation in which 2 of 3 leaflets of the aortic valve are fused to form a two-leaflet valve. BAV is the most common congenital cardiovascular malformation in adults, with an incidence of $1.37 \%$ in the general population. ${ }^{1}$ Patients with BAV are at increased risk for aortic valve diseases and aortopathies, including aortic dilatation, aneurysm and dissection, compared to the population with tricuspid aortic valve (TAV). Larsons and Edwards reported that the risk of aortic dissection is 9-fold higher with BAV compared with TAV. ${ }^{2}$ The American College of Cardiology and American Heart Association (ACC/AHA) Guidelines in 2016 specify surgical intervention for an ascending aorta of $6.0 \mathrm{~cm}$ for TAV cases, $5.5 \mathrm{~cm}$ for BAV cases designated as class I, and $4.5 \mathrm{~cm}$ for cases requiring aortic valve replacement (AVR) for aortic stenosis (AS) or aortic insufficiency, which are designated as class IIa. ${ }^{3}$

There are 2 hypotheses that are not mutually exclusive for the association of BAV and aortopathies. One is the effect of hemodynamic abnormalities caused by turbulent

\section{Editorial p 2470}

blood flow due to the abnormal structure of BAV. The turbulence is evidenced by electrocardiogram-gated computed tomographic angiography ${ }^{4}$ and spin echo magnetic resonance imaging, ${ }^{5}$ both suggesting increased wall stress in the ascending aorta in BAV. The second hypothesis is the fragility of the ascending aorta due to abnormal morphogenesis associated with BAV. Many reports have shown reduced collagen, elastic fibers and smooth muscle cells in the ascending aorta in patients with BAV. ${ }^{6-9}$ Biner et al found significantly lower aortic distensibility using echocardiogram and blood pressure parameters, ${ }^{10}$ and Pasta et al showed the fragility of the ascending aorta in BAV through direct measurements of mechanical dissection properties of specimens from patients with BAV or TAV. ${ }^{11}$ Borger et al suggested that dilatation of the ascending aorta is likely to occur even after AVR for BAV when the diameter of the ascending aorta is $\geq 45 \mathrm{~mm},{ }^{12}$ suggesting the presence of the fragility of the aorta after correction of

Received January 22, 2018; revised manuscript received June 13, 2018; accepted June 26, 2018; released online August 8, 2018 Time for primary review: 24 days

Division of Cardiovascular Surgery, Department of Surgery (Y.H., T.S., K.T., T.T., K.A., S.T., H.T.), Division of Cardiovascular Medicine, Department of Internal Medicine (Y.F.), Kurume University School of Medicine, Kurume; Cardiovascular Research Institute, Kurume University, Kurume (H.A.), Japan

Mailing address: Hiroki Aoki, MD, PhD, Cardiovascular Research Institute, Kurume University, 67 Asahimachi, Kurume 830-0011, Japan. E-mail: haoki@med.kurume-u.ac.jp

ISSN-1346-9843 All rights are reserved to the Japanese Circulation Society. For permissions, please e-mail: cj@j-circ.or.jp 


\begin{tabular}{lcc} 
Table 1. Case Numbers in Each Analysis Modality \\
\multicolumn{4}{c}{ BAV } & TAV \\
Enrollment & $\# 1-\# 9$ & $\# 1-\# 13$ \\
Transcriptome & $\# 1-\# 9$ & $\# 2, \# 3, \# 4, \# 6, \# 8, \# 9, \# 11$ \\
Immunohistochemistry & $\# 1-\# 9$ & $\# 1-\# 5, \# 7-\# 13$ \\
\hline
\end{tabular}

BAV, bicuspid aortic valve; TAV, tricuspid aortic valve. For transcriptome analysis, RNA samples with insufficient quality or quantity were excluded. For histological analysis, the aortic wall specimens without 3 layers, namely: intima, media and adventitia, were excluded.

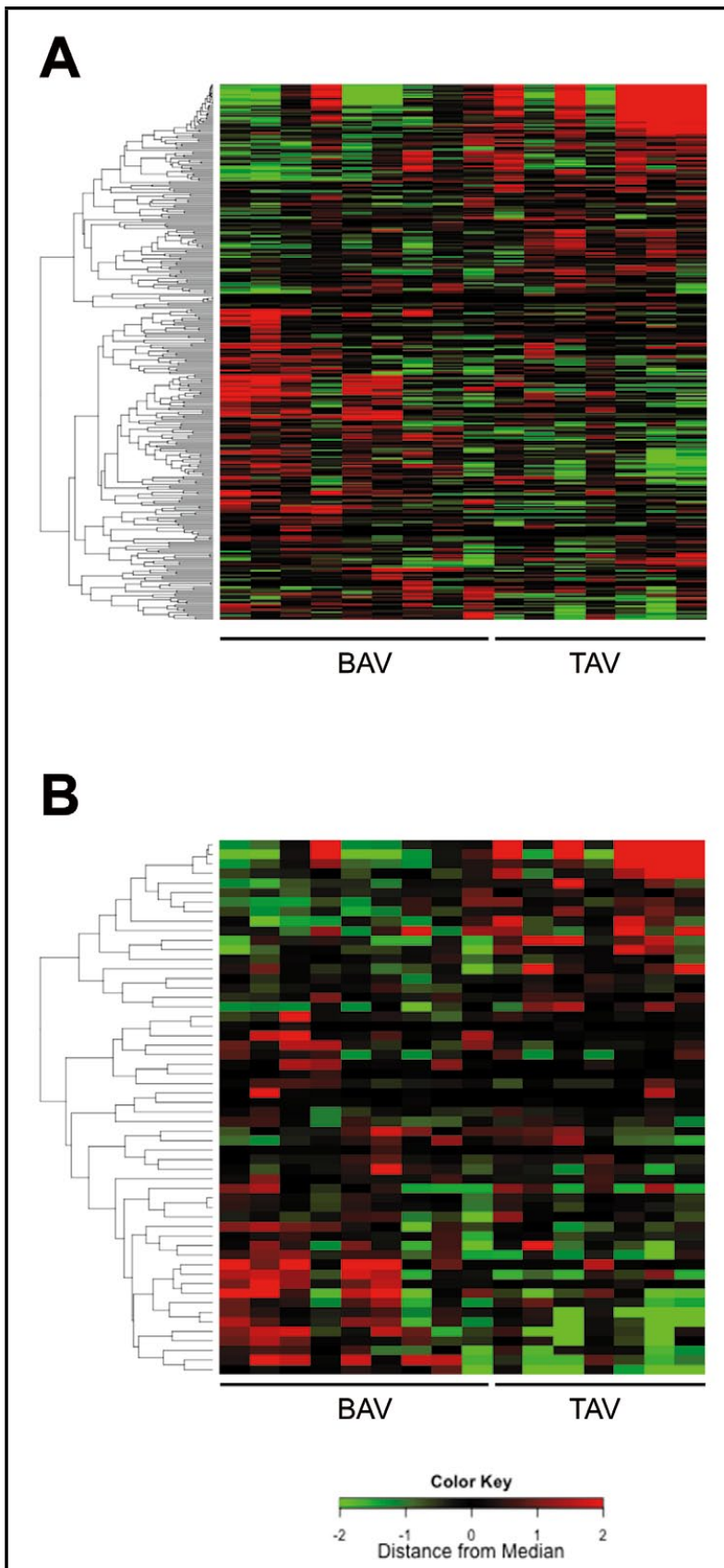

Figure 1. Heat maps for functional annotation clusters. The heat maps show the results of hierarchical clustering analysis for the functional annotation clusters \#1 (A) and \#2 (B). After log conversion of the signal value, the distance from the median is presented in green, black, and red when lower, intermediate, and higher than other samples respectively.
BAV-associated turbulent flow. With regard to the molecular basis of BAV and aortopathy, NOTCH1 is of interest because a mutation in this gene was the first to be associated with familial BAV and aortopathy in humans ${ }^{13}$ and aortopathy in mice. ${ }^{14}$ Mutations of other genes are also associated with BAV, including GATA5,15 TGFBR1, TGFBR $2^{16}$ and NKX2.5. ${ }^{17}$ However, the molecular basis of aortopathy associated with non-familial BAV remains elusive. In this study, we examined the molecular characteristics of the ascending aorta in patients with BAV and TAV.

\section{Methods}

\section{Human Subjects}

All protocols that involved human specimens were approved by the Institutional Review Board at Kurume University Hospital, and all samples were obtained with written informed consent from the patients. The subjects were 22 consecutive patients who underwent AVR for AS at Kurume University Hospital, including 9 with BAV and 13 with TAV, from July 2013 to February 2014 (Table 1). Patients with aortic insufficiency, a re-operated case, those with clinical signs of connective tissue diseases such as Marfan syndrome and Ehlers-Danlos syndrome, and those receiving hemodialysis were excluded. The specimens of the ascending aorta were collected during surgery and subjected to the analyses (Figure 1). All patients underwent initial chest computed tomography (CT) and trans-thoracic echocardiography at our hospital or their regular hospital. The maximal diameter of the ascending aorta and the diameters of the sinus of Valsalva and sinotubular junction (STJ) were determined on axial chest CT. The ascending aortic wall was partially resected from the incised aortotomy region. In patients treated with aortic root replacement, a portion of the ascending aorta incised by aortectomy was collected. A full-thickness specimen of the anterior wall of the ascending aorta in the aortotomy region $(1.5 \mathrm{~cm}$ peripheral to the STJ) was collected during AVR or aortic root replacement. The specimen was divided immediately after sampling in the operating room, and stored in RNAlater for transcriptome analysis and in 3.7\% formaldehyde/ phosphate-buffered saline for histopathology.

\section{Transcriptome Analysis}

Total RNA was isolated from the aortic walls using TRIzol Reagent and liquid nitrogen, and purified using a SV Total RNA Isolation System (Promega). RNA samples were quantified using a ND-1000 spectrophotometer (NanoDrop Technologies) and the quality was confirmed with an Experion System (Bio-Rad Laboratories). RNA samples from $6 \mathrm{TAV}$ patients were with insufficient quality or quantity, and were excluded from the transcriptome analysis (Table 1). cRNA was amplified, labeled, and hybridized to a 60-mer oligomicroarray (SurePrint G3 Human GE 8x60K Microarray; Agilent). All hybridized microarray slides were scanned by an Agilent scanner. Relative hybridization intensities and background hybridization values were calculated using Agilent Feature Extraction Software. The raw data of the transcriptome analysis has been deposited to Gene Expression Omnibus, with the accession number GSE83675. The raw signal intensities of all samples were $\log _{2}$-transformed and normalized by a quantile algorithm with the 'preprocessCore' library package in Bioconductor software. Probes with detection P-values $<0.01$ in all samples (excluding control probes) were selected and used 


\begin{tabular}{|c|c|c|c|}
\hline Item & $\begin{array}{l}\text { BAV } \\
(n=9)\end{array}$ & $\begin{array}{c}\text { TAV } \\
(n=13)\end{array}$ & P-value \\
\hline Age (years) & $75.78 \pm 7.68$ & $79.2 \pm 5.57$ & 0.170 \\
\hline \multicolumn{4}{|l|}{ Sex, n (\%) } \\
\hline Male & $5(55.6 \%)$ & $4(30.8 \%)$ & \\
\hline Female & $4(44.4 \%)$ & $9(69.2 \%)$ & 0.256 \\
\hline Height (cm) & $155.04 \pm 7.99$ & $147.57 \pm 11.75$ & 0.166 \\
\hline Weight (kg) & $48.47 \pm 11.31$ & $50.15 \pm 19.47$ & 0.894 \\
\hline $\mathrm{BSA}\left(\mathrm{m}^{2}\right)$ & $1.44 \pm 0.18$ & $1.39 \pm 0.30$ & 0.547 \\
\hline \multicolumn{4}{|l|}{ Echographic parameters } \\
\hline $\operatorname{AVA}\left(\mathrm{cm}^{2}\right)$ & $0.57 \pm 0.33$ & $0.59 \pm 0.21$ & 0.841 \\
\hline $\mathrm{AVA} / \mathrm{BSA}\left(\mathrm{cm}^{2} / \mathrm{m}^{2}\right)$ & $0.40 \pm 0.23$ & $0.42 \pm 0.11$ & 0.841 \\
\hline Sinus of Valsalva (mm) & $39.11 \pm 9.49$ & $30.23 \pm 3.76$ & 0.00117 \\
\hline Sinotubular junction (mm) & $33.13 \pm 12.03$ & $23.54 \pm 3.29$ & 0.00283 \\
\hline Ascending aorta $(\mathrm{mm})$ & $43.56 \pm 9.96$ & $33.00 \pm 4.11$ & 0.00136 \\
\hline Ascending aorta/BSA $\left(\mathrm{mm} / \mathrm{m}^{2}\right)$ & $30.53 \pm 8.82$ & $23.95 \pm 3.49$ & 0.0299 \\
\hline \multicolumn{4}{|l|}{ Preoperative complications, $\mathrm{n}(\%)$} \\
\hline Hypertension & $5(55.6 \%)$ & $13(100.0 \%)$ & 0.0125 \\
\hline Diabetes mellitus & $5(55.6 \%)$ & $3(23.1 \%)$ & 0.256 \\
\hline Smoking & $4(44.4 \%)$ & $4(30.8 \%)$ & 0.522 \\
\hline \multicolumn{4}{|l|}{ Medications, n (\%) } \\
\hline ACE-I and/or ARB & $3(33.3 \%)$ & $8(61.5 \%)$ & 0.204 \\
\hline$\beta$-blocker & $0(0.0 \%)$ & $3(23.1 \%)$ & 0.130 \\
\hline Calcium channel blocker & $1(11.1 \%)$ & $9(69.2 \%)$ & 0.00854 \\
\hline Statins & $2(22.2 \%)$ & 7 (53.8\%) & 0.147 \\
\hline
\end{tabular}

Basic characteristics of the patients are shown for those with BAV and TAV. ACE-I, angiotensin-converting enzyme inhibitor; ARB, angiotensin II receptor blocker; AVA, aortic valve area; BAV, bicuspid aortic valve; BSA, body surface area; TAV, tricuspid aortic valve.

to identify differentially expressed genes. The Linear Models for Microarray Analysis (limma) package in the Bioconductor software was then used to obtain genes, based on criteria of a limma P-value $<0.05$ and an absolute logfold-change $(|\log \mathrm{FC}|)>1$ between control and experiment samples. Functional annotation clusters were obtained using the Database for Annotation, Visualization, and Integrated Discovery (DAVID, https://david.ncifcrf.gov/), with the Gene Ontology terms set to GOTERM_BP_FAT, GOTERM_CC_FAT and GOTERM_MF_FAT.

\section{Immunohistochemical Staining}

Immunohistochemical staining was performed using antibodies for pAKT (S473; Cell Signaling Technology \#4060, x150 dilution) or AKT (Cell Signaling Technology \#9272, x300 dilution) and CSA II Biotin-free Tyramide Signal Amplification System (DAKO Cytomation \#K1497). Regions in which the 3-layer structure of the aorta could be confirmed were evaluated. The medial layer, as identified by the elastic lamellar structure, was divided into 3 zones, namely: inner, middle and outer zones, with equal thickness to evaluate the local pAKT or AKT expression. The pAKT- or AKT-positive area per unit area was measured in 3 zones of the medial layer of the ascending aorta using ImagePro Plus Ver. 7.0 (Media Cybernetics).

\section{Immunofluorescence Staining and Imaging Cytometry}

Immunofluorescence staining was performed using antibodies for pAKT (S473; Cell Signaling Technology \#4060, x20 dilution) with a signal amplification kit (AlexaFluor 488 Tyramide SuperBoost Kit; ThermoFisher Scientific

Table 3. Functional Annotation Clusters With High
Enrichment Scores
Annotation Cluster 1
Enrichment Score: 2.9278006644191006
Term
GO:0031226 intrinsic to plasma membrane
GO:0005887 integral to plasma membrane
GO:0044459 plasma membrane part
GO:0005886 plasma membrane
Annotation Cluster 2
Enrichment Score: 2.689251769161405
Term
GO:0007169 transmembrane receptor protein tyrosine
kinase signaling pathway
GO:0004714 transmembrane receptor protein tyrosine
kinase activity
GO:0007167 enzyme linked receptor protein signaling
pathway

Functional annotation clusters with highest enrichment scores, as obtained by Database for Annotation, Visualization and Integrated Discovery (DAVID) analysis, are shown. GO, gene ontology.

\#B40922), AlexaFluor 647-conjugated smooth muscle $\alpha$-actin antibody ( $\alpha$ SMA; Novus Biologicals \#NBP234522AF647, x30 dilution) and nuclear staining with 4',6-diamidino-2-phenylindole (DAPI). Imaging cytometric analyses were performed for the inner, middle and outer zones of the medial layer in the same way as that 


\begin{tabular}{|c|c|c|c|c|c|c|c|}
\hline \multirow[t]{2}{*}{ Gene symbol } & \multirow[t]{2}{*}{ Description } & \multicolumn{2}{|c|}{ BAV vs. TAV } & \multicolumn{2}{|c|}{$\begin{array}{c}\text { BAV; Dilation } \\
(+) \text { vs. (-) }\end{array}$} & \multicolumn{2}{|c|}{$\begin{array}{l}\text { Dilation (-); } \\
\text { BAV vs. TAV }\end{array}$} \\
\hline & & $P$ value & Ratio & $P$ value & Ratio & $P$ value & Ratio \\
\hline RAPGEF1 & Rap guanine nucleotide exchange factor 1 & $0.0047^{*}$ & 3.38 & 0.6138 & 1.33 & $0.0271^{*}$ & 2.97 \\
\hline GREM2 & Gremlin 2, cysteine knot superfamily & $0.0314^{*}$ & 3.23 & 0.6097 & 1.50 & 0.0633 & 2.70 \\
\hline REPS2 & RALBP1 associated Eps domain containing 2 & $0.0078^{*}$ & 3.12 & 0.8672 & 1.07 & $0.0472^{*}$ & 3.03 \\
\hline FGFR3 & Fibroblast growth factor receptor 3 & $0.0296^{*}$ & 2.88 & 0.9517 & 0.95 & 0.0546 & 2.94 \\
\hline PTPRJ & Protein tyrosine phosphatase, receptor type, J & $0.0295^{\star}$ & 2.45 & 0.0359 & 1.87 & 0.2081 & 1.86 \\
\hline FGF18 & Fibroblast growth factor 18 & $0.0342^{*}$ & 2.39 & 0.4634 & 1.49 & 0.1405 & 2.00 \\
\hline FGF9 & Fibroblast growth factor 9 & $0.0499^{*}$ & 2.32 & 0.2131 & 1.92 & 0.2500 & 1.73 \\
\hline KIT & Proto-oncogene tyrosine-protein kinase Kit (c-kit) & $0.0155^{\star}$ & 2.27 & 0.6055 & 1.17 & 0.0778 & 2.12 \\
\hline FKBP1A & FK506 binding protein $1 \mathrm{~A}$ & $0.0320^{*}$ & 2.21 & 0.2520 & 1.58 & 0.1801 & 1.80 \\
\hline EIF2AK3 & Eukaryotic translation initiation factor 2- $a$ kinase 3 & 0.1834 & 1.52 & $0.0171^{*}$ & 2.77 & 0.9129 & 0.97 \\
\hline MUSK & Muscle, skeletal, receptor tyrosine kinase & 0.3568 & 1.34 & $0.0458^{*}$ & 2.09 & 0.9208 & 0.96 \\
\hline GRIK2 & Glutamate receptor, ionotropic, kainate 2 & 0.5493 & 1.17 & $0.0066^{*}$ & 0.43 & 0.0745 & 1.71 \\
\hline EPHB2 & EPH receptor B2 & 0.9854 & 1.00 & $0.0076^{*}$ & 2.15 & ND & ND \\
\hline ROR2 & Receptor tyrosine kinase-like orphan receptor 2 & 0.8638 & 0.93 & $0.0417^{\star}$ & 2.45 & ND & ND \\
\hline BCAR1 & Breast cancer anti-estrogen resistance 1 & $0.0092^{*}$ & 0.47 & ND & ND & 0.1079 & 0.59 \\
\hline PML & Promyelocytic leukemia protein & $0.0295^{\star}$ & 0.55 & 0.0275 & 0.46 & 0.3641 & 0.78 \\
\hline FGFR4 & Fibroblast growth factor receptor 4 & $0.0020^{*}$ & 0.48 & 0.5928 & 0.84 & $0.0223^{*}$ & 0.52 \\
\hline LTK & Leukocyte receptor tyrosine kinase & $0.0296^{\star}$ & 0.47 & 0.0956 & 0.54 & 0.2023 & 0.62 \\
\hline BAIAP2 & BAl1-associated protein 2 & $0.0137^{\star}$ & 0.47 & 0.5856 & 0.89 & 0.0743 & 0.49 \\
\hline TSC2 & Tuberous sclerosis 2 & $0.0058^{*}$ & 0.41 & 0.9643 & 1.02 & $0.0241^{*}$ & 0.40 \\
\hline VEGFA & Vascular endothelial growth factor $A$ & $0.0340^{*}$ & 0.37 & 0.0703 & 2.37 & $0.0207^{\star}$ & 0.25 \\
\hline IL31RA & Interleukin 31 receptor $A$ & $0.0089^{*}$ & 0.36 & 0.2426 & 1.64 & $0.0088^{*}$ & 0.29 \\
\hline NIN & Ninein & $0.0084^{*}$ & 0.25 & 0.3932 & 1.38 & $0.0211^{*}$ & 0.22 \\
\hline EGFR & Epidermal growth factor receptor & $0.0124^{\star}$ & 0.13 & 0.3619 & 2.00 & $0.0206^{\star}$ & 0.09 \\
\hline CD3E & CD3e molecule, epsilon & $0.0015^{\star}$ & 0.12 & 0.3951 & 1.48 & $0.0057^{*}$ & 0.10 \\
\hline
\end{tabular}

The list indicates the official symbols and the description of the genes in cluster \#2. $\mathrm{P}$ values and ratios are shown for the indicated comparisons. * $\mathrm{P}$ values indicate $\mathrm{P}<0.05$. ND, not detected; BAV, bicuspid aortic valve; TAV, tricuspid aortic valve.

conducted in the immunohistochemical study, using ArrayScan XTI (ThermoFisher Scientific) and FlowJo Software (FlowJo LLC). In the imaging cytometric analyses, each cell was identified by nuclear DAPI staining and arbitrarily gated to separate the positive (smooth muscle cells) and negative (non-smooth muscle cells) populations for $\alpha$ SMA signal surrounding the nucleus. The gate for the signal intensity of pAKT was also determined arbitrarily. These gates were set constant throughout the analysis.

\section{Statistical Analysis}

Data are expressed as means \pm standard errors. Statistical analyses were performed with GraphPad PRISM 5 (GraphPad Software). When the data passed the D'Agostino and Pearson normality test and Bartlett's test for equal variances, we performed an unpaired t-test for 2 groups and a one-way ANOVA to compare 3 or more groups, followed by Bonferroni's multiple comparison test. For non-normal data distributions, we performed the MannWhitney U-test for 2 groups and the Kruskal-Wallis test for more than 2 groups, followed by Dunn's multiple comparison test. Statistical significance was indicated by a $\mathrm{P}$-value $<0.05$.

\section{Results}

Patient Characteristics

Patient characteristics, severity of aortic valve stenosis, aortic diameter, comorbidities, and oral drugs were surveyed in 9 patients with BAV and 13 with TAV (Table 2). The proportion of male patients was $55.6 \%$ and $30.8 \%$, mean ages were $75.78 \pm 7.68$ and $79.2 \pm 5.57$ years, and the mean body surface area (BSA) was $1.44 \pm 0.18$ and $1.39 \pm 0.30 \mathrm{~m}^{2}$ in the BAV and TAV groups respectively. There was no significant difference in these parameters between the 2 groups. The mean AVA/BSA was $0.57 \pm 0.33$ and $0.59 \pm 0.21 \mathrm{~cm}^{2}$ in the BAV and TAV groups respectively. The BAV group had significantly larger mean diameters at the sinus Valsalva $(39.11 \pm 9.49$ vs. $30.23 \pm 3.76 \mathrm{~mm}$, $\mathrm{P}<0.05)$, at the STJ $(33.13 \pm 12.03$ vs. $23.54 \pm 3.29 \mathrm{~mm}$, $\mathrm{P}<0.05)$, and at the ascending aorta/BSA $(30.53 \pm 8.82$ vs. $\left.23.95 \pm 3.49 \mathrm{~mm} / \mathrm{m}^{2}, \mathrm{P}<0.05\right)$.

\section{Transcriptome Analysis of Ascending Aortic Walls}

To obtain an insight into the difference between BAV and TAV, we performed transcriptome analysis. The total RNA extracted from the aortic samples were subjected to the initial quality check, in which 9 cases in the BAV group and 7 cases in the TAV group passed. The genes with altered expressions between the BAV and TAV regardless of aortic dilation, the BAV with and without dilation, and BAV and TAV without aortic dilation were defined as those with an absolute log-fold-change of more that 2 and a P-value of less than 0.05 . Among the 41,628 probes on the DNA microarray, 843 probes fulfilled this criteria, and they were subjected to the functional annotation analysis 
using DAVID. ${ }^{18}$ The annotation cluster with the highest enrichment score (2.928) was defined with gene ontology terms as "intrinsic plasma membrane" and "integral to plasma membrane", which comprised 342 probes (Table 3 , Figure 1A). The annotation cluster with the second highest enrichment score (2.689) was defined with gene ontology terms as "transmembrane receptor protein tyrosine kinase signaling pathway", which comprised 56 probes (Table 3 , Figure 1B) representing 25 genes (Table 4). These results suggested that transmembrane signaling may be altered in the aortic pathology associated with BAV, among which the receptor tyrosine kinase (RTK) pathway may be important.

To explore the significance of the different gene expression profiles between TAV and BAV, we focused on the second annotation cluster that was related to the RTK pathway, because it may have a functional relevance to aortopathies. The RTK-related molecules in the annotation cluster \#2 were in different classes, such as receptors, ligands and signal modulators, and they were either induced or suppressed in BAV samples (Table 4). Therefore, it seemed that the RTK pathway is activated in concert to make a complex network, although it was not obvious whether the overall RTK pathway was activated or suppressed in BAV compared to TAV.

\section{AKT Activation in Ascending Aortic Walls}

We next examined the histology of the ascending aortic walls. Histochemical study with elastica van Gieson (EVG) and hematoxylin and eosin (H\&E) staining showed an orderly arranged structure of elastic lamellae in the medial layer both in TAV and BAV samples without obvious inflammation or tissue destruction (Figure 2). To obtain the insight into the activation status of the RTK pathway, we stained aortic samples for total AKT, the main signaling molecule in this pathway, and for the activated (phosphorylated) AKT (pAKT). Among the aortic wall samples obtained from all of the cases, those with intact intima, media and adventitial layers were analyzed. Accordingly, 9 cases in the BAV group and 12 cases in the TAV group were observed. Among them, 4 cases of BAV cases showed the dilation of the ascending aorta more than $45 \mathrm{~mm}$, and none of the TAV cases showed dilation of the ascending aorta. Within the 3 layers of aortic structure, we focused on the medial layer, because it is the main load-bearing structure that shows pathological changes in various aortopathies. Immunohistochemical pAKT staining showed activation (phosphorylation) of AKT mainly in the medial layer, whereas total AKT was found throughout the aortic walls. We divided the medial layer into 3 zones with equal thickness, namely: inner, medial and outer medial zones, to examine whether pAKT revealed differences in the expression within the medial layer (Figure 3). Two-way ANOVA analysis for the valvular status (TAV or BAV) and the medial zones (inner, middle or outer zone) within the medial layer revealed that the presence of BAV significantly contributed to the difference in $\mathrm{pAKT}$-positive area $(\mathrm{P}=0.0003)$, whereas the zones did not $(\mathrm{P}=0.052)$. When TAV and BAV samples were compared between matched zones, BAV showed a higher pAKT signal in the outer zone $(\mathrm{P}<0.01)$. When we examined the expression of $\mathrm{pAKT}$ in the $\mathrm{BAV}$ group, the presence/absence of dilation in the ascending aorta $(\mathrm{P}=0.909$, Figure 3B) or the zones within the medial layer $(\mathrm{P}=0.262)$ did not show significant contribution. Two-way ANOVA analysis of the total AKT-positive area

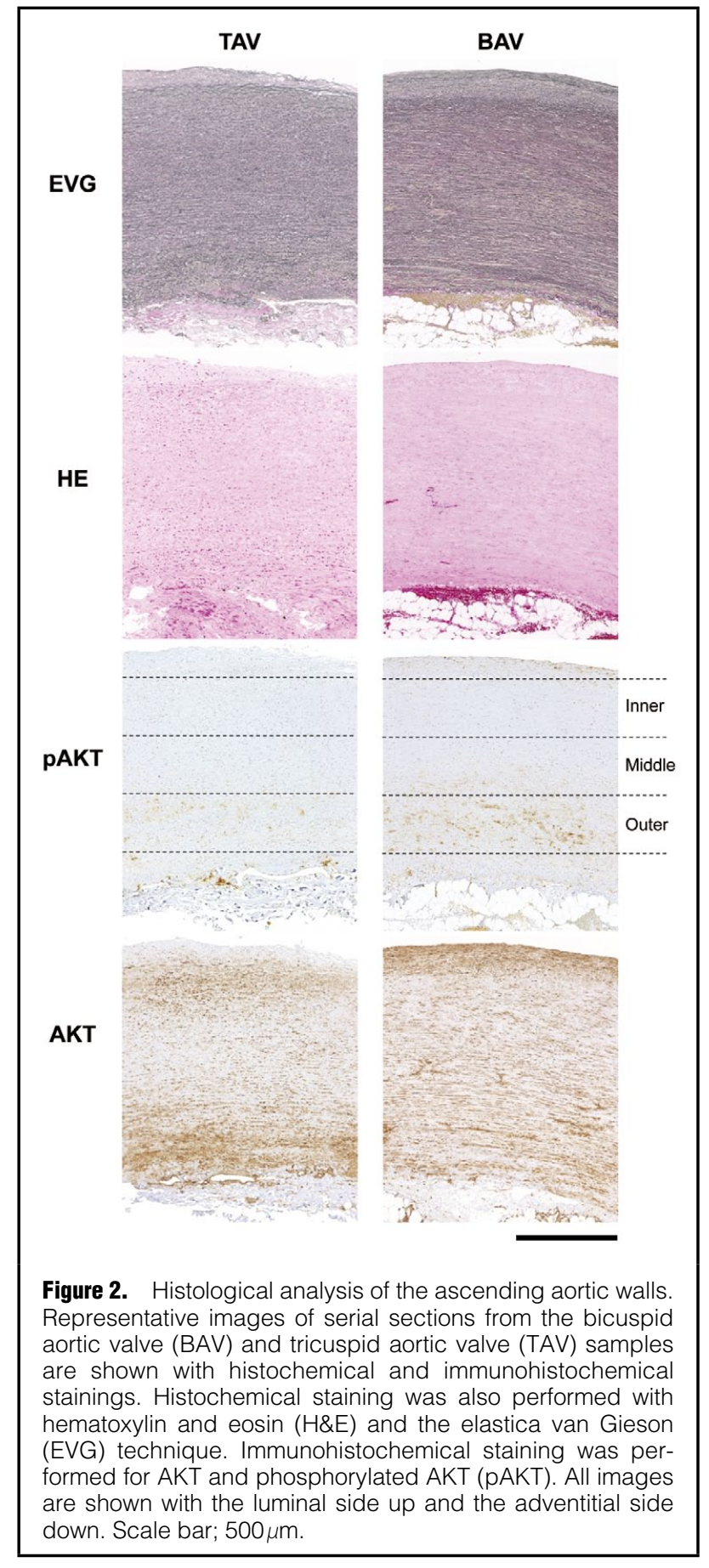

revealed that both of the valvular status (TAV or BAV; $\mathrm{P}=0.024)$ and the zones $(\mathrm{P}=0.0004)$ showed significant contribution to AKT expression. These results indicated that AKT was activated in the BAV ascending aorta, regardless of the aortic dilation, compared to the TAV aorta. In addition, the patterns of the expression and the activation of $\mathrm{AKT}$ are different, indicating that AKT activation is not merely due to the higher AKT protein expression.

\section{Cell Types With Activated AKT in the Aortic Medial Layer}

To further characterize the AKT activation in the aortic medial for the cell types, we performed double immuno- 


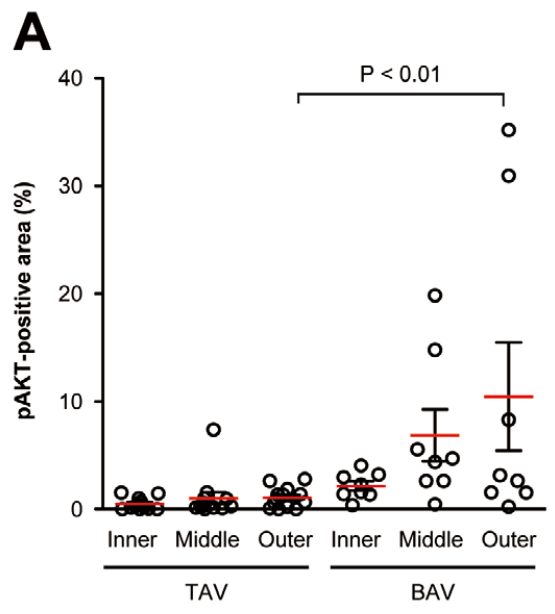

C

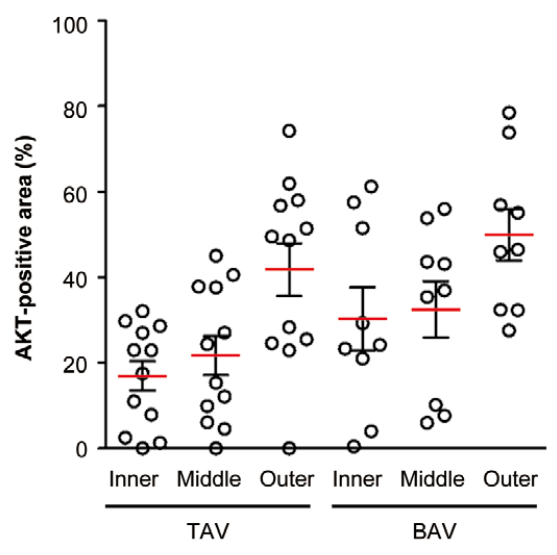

B

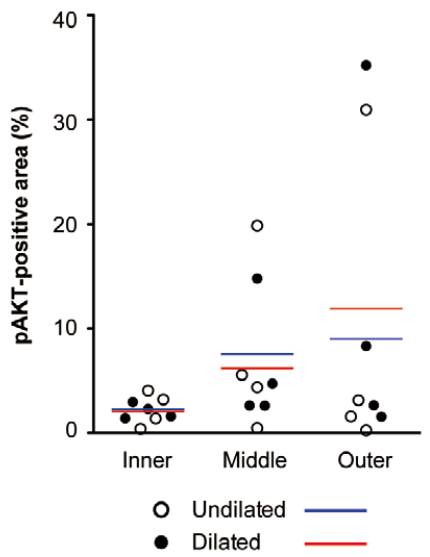

Figure 3. pAKT-positive area in the aortic wall samples. (A) The graph shows the pAKT-positive area in the inner, middle, and outer zones of the medial layer in the aortic samples with tricuspid aortic valve (TAV) and bicuspid aortic valve (BAV). Red and black bars indicate means and standard errors respectively. (B) The graph shows the pAKT-positive area in the inner, middle, and outer zones of the medial layer in the aortic samples with BAV. Open symbols and blue bars indicate individual data and means respectively, for samples without ascending aorta dilation. Closed symbols and red bars indicate those samples with ascending aorta dilation, as defined by the diameters of more than $45 \mathrm{~mm}$. (C) The graph shows the AKT-positive area in the inner, middle, and outer zones of the medial layer in the aortic samples with TAV and BAV. Red and black bars indicate means and standard errors respectively. fluorescence staining for pAKT and smooth muscle $\alpha$-actin ( $\alpha$ SMA), a marker of smooth muscle cells (Figure 4). The immunofluorescence study revealed that the pAKT signal mostly overlapped with part of the $\alpha$ SMA staining (Figure 4A). We performed imaging cytometric analysis, in which a single cell was identified by DAPI nuclear staining; the cell identity was defined by the $\alpha$ SMA signal intensity surrounding the nuclei, and the pAKT signal intensities were quantitated at the single cell level. In the scattergram, where $\mathrm{X}$ - and $\mathrm{Y}$-axes indicate the signal intensities of $\alpha$ SMA and pAKT respectively, the pAKT-positive cells were located in the upper right quadrant (Figure 4B). These findings indicated that the pAKT-positive cells were smooth muscle cells in the aortic medial layer.

\section{Discussion}

In this study, transcriptome analysis showed enrichment of genes encoding molecules related to the RTK pathway in BAV aorta compared to TAV aorta. These molecules included growth factors, their receptors, and mammalian target of rapamycin (mTOR) pathway molecules. At the protein level, the activation of AKT, an essential signal mediator in the RTK pathway, was significantly higher in the aortic medial layer with BAV than that with TAV.
Notably, AKT was activated in BAV regardless of the extent of aortic dilation, suggesting that its activation was not the consequence of the aortic dilation. Within the aortic wall with BAV, AKT tended to be more active on the adventitial side of the medial layer, suggesting the heterogeneity of the local environment for the RTK pathway and AKT activation.

The role of RTK/AKT in aortopathies is currently poorly understood. RTKs are transmembrane proteins that convert extracellular stimulation by growth factors into an intracellular signal, with activation of phosphoinositide 3-kinase (PI3K), AKT and mTOR. Because this pathway regulates cell growth and differentiation and is involved in tissue repair, the activation of RTK/AKT can be an adaptive response to promote the repair of fragile and injured aortic wall due to the genetic and hemodynamic abnormalities associated with BAV. ${ }^{6-9}$ However, because cell growth and proliferation is central to tissue remodeling, it is also possible that the RTK/AKT pathway may be involved in the expansion of the aortic diameter that is frequently associated with BAV. In this regard, our data indicated that AKT activation in the aortic wall tissue with BAV occurred regardless of aortic diameter, suggesting that AKT activation may precede aortic dilation. Interestingly, cell culture studies showed mTOR, a signaling 
A


BAV
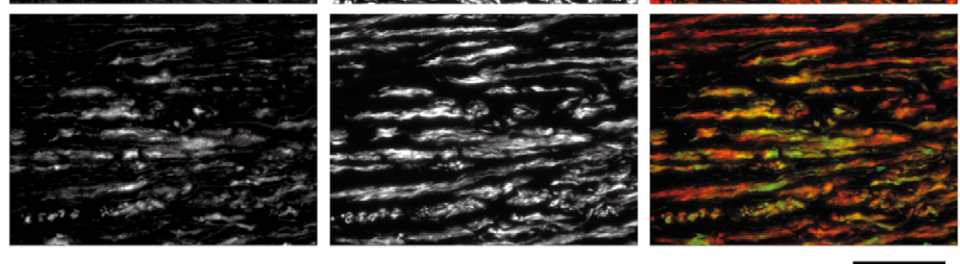

B
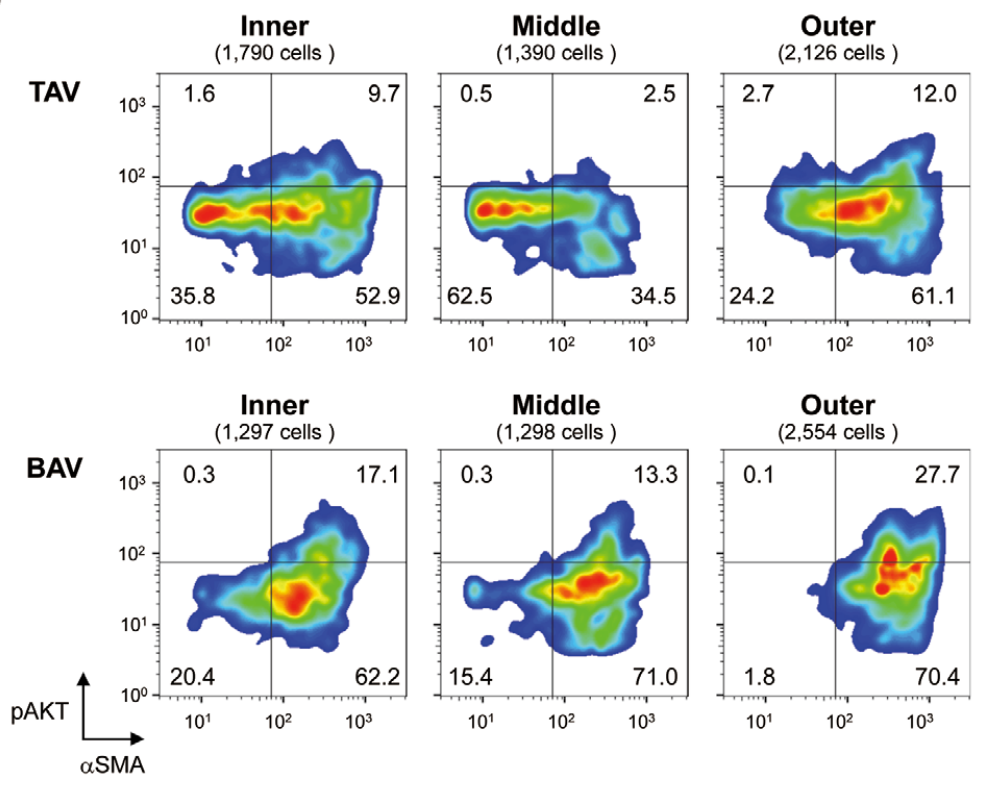

Figure 4. Expression of pAKT in the medial layer of the aorta. (A) Representative images are shown for immunofluorescence staining for pAKT and smooth muscle a-actin (aSMA) in the outer zone of the aortic wall samples from tricuspid aortic valve (TAV) or bicuspid aortic valve (BAV) patients. The merged images show PAKT staining in green and aSMA staining in red. Scale bar; $100 \mu \mathrm{m}$. (B) The results of the imaging cytometric analysis are shown by scattergrams, where the $\mathrm{X}$-axis and $\mathrm{Y}$-axis indicate the signal intensities of aSMA and pAKT respectively. The gates for the positive/negative signal of aSMA and pAKT are arbitrarily determined and set consistent throughout the observational groups, namely: inner, middle, outer zones of the TAV or BAV samples. The numbers of the counted cells are shown in parentheses for each zone of the TAV or BAV samples. The numbers in quadrants indicate the percentages of the cell population.

module under the RTK/AKT pathway, is involved in proliferation of aortic smooth muscle cells, ${ }^{19,20}$ and animal studies demonstrated that abnormal cell proliferation is associated with the aortopathies in elastin-deficient mice. ${ }^{21}$ Future studies should be directed at whether and how the RTK pathway is involved in the aortopathies associated with BAV.

Also of interest is how the RTK pathway is activated in the aortic tissue with BAV. Regarding the RTKs and their ligands, our transcriptome analysis indicated that FGFR3, FGF18, FGF9 and KIT were induced in BAV tissue. Because these molecules are implicated in myofibroblast differentiation ${ }^{22,23}$ and remodeling of the aorta, ${ }^{24}$ they may well be involved the activation of AKT, as observed in this study and the aortopathies. Another potential mechanism for the activation of the RTK pathway is the ligandindependent mechanism. For example, NOTCH1 has been shown to repress the PI3K/AKT pathway in cell culture experiments, ${ }^{25}$ and the loss-of-function mutation of NOTCH1 is a causative mutation for BAV.13 Therefore, activation of the RTK pathway, including AKT, may be involved with aortopathies due to the loss-of-function mutation of NOTCH. These previous reports, together with our current findings, suggest that RTK/AKT activation may participate in the pathogenesis of aortopathies associated with BAV. Further studies are required to decipher the molecular basis of aortopathies with regard to the RTK/AKT pathway in BAV.

\section{Study Limitations}

We recognize several limitations of this study. Due to the small number of patients, we were unable to extract the factors that might affect AKT activation other than the presence of BAV. Because this study was the initial attempt to identify the molecular pathway that is characteristic to $\mathrm{BAV}$, we did not perform an in-depth investigation for the 
molecular events under the RTK/AKT pathway or the mechanism for RTK/AKT activation. Also, we did not examine the culprit genes for BAV. ${ }^{26}$ Therefore, the patients in this study are likely to be genetically heterogeneous, and the relationship between the genetic abnormalities and the RTK/AKT pathway remains to be investigated in the future. Regarding the AKT expression and activity, a previous study reported the decreased AKT and pAKT in BAV ascending aortic aneurysms (AsAA) compared to TAV AsAA. ${ }^{27}$ Apparent discrepancy between the current findings and the previous report may be because the previous report analyzed only AsAA; aortic diameters were $5.5 \pm 0.7 \mathrm{~cm}$ and $4.9 \pm 0.3 \mathrm{~cm}$ for TAV and BAV respectively, whereas our samples were $3.3 \pm 0.4 \mathrm{~cm}$ (TAV) and $4.4 \pm 1.0 \mathrm{~cm}$ (BAV), obtained from aortic valve stenosis patients. It remains to be clarified how aortic diameters or the presence of aortic valve stenosis affects the AKT expression and activity. In this regard, analysis of normal aorta without BAV or AsAA would provide the baseline for the expression and activity of AKT. However, normal aorta without aortic diseases was not available, which is another important limitation of the current study.

\section{Conclusions}

In conclusion, our findings revealed higher activation of the RTK/AKT pathway in aortae with BAV, which may reflect the abnormal tissue response and contribute to the structural abnormality of the aorta in these patients. Further research is required to clarify how the RTK/AKT pathway is activated in the aortic tissue with BAV, to define the involvement of the RTK/AKT pathway in the aortopathies associated with $\mathrm{BAV}$, and ultimately to establish the diagnostic and therapeutic significance of the findings in this study.

\section{Funding}

This work was supported, in part, by JSPS KAKENHI JP15K19929 (Y.H.) and JSPS KAKENHI 16H05428 (H.A.).

\section{References}

1. Hoffman J. The global burden of congenital heart disease. Cardiovasc J Afr 2013; 24: 141-145.

2. Larson EW, Edwards WD. Risk factors for aortic dissection: A necropsy study of 161 cases. Am J Cardiol 1984; 53: 849-855.

3. $2010 \mathrm{ACCF} / \mathrm{AHA} / \mathrm{AATS} / \mathrm{ACR} / \mathrm{ASA} / \mathrm{SCA} / \mathrm{SCAI} / \mathrm{SOR} / \mathrm{STS} / \mathrm{SVM}$ Guidelies for the Diagnosis and Management of Patients With Thoracic Aortic Disease Representative Members, Hiratzka LF, Creager MA, Isselbacher EM, Svensson LG, 2014 AHA/ACC Guidelin for the Management of Patients With Valvular Heart Disease Representative Members, et al. Surgery for aortic dilatation in patients with bicuspid aortic valves: A statement of clarification from the American College of Cardiology/American Heart Association Task Force on Clinical Practice Guidelines. $J$ Thorac Cardiovasc Surg 2016; 151: 959-966.

4. Nathan DP, Xu C, Plappert T, Desjardins B, Gorman JH 3rd, Bavaria JE, et al. Increased ascending aortic wall stress in patients with bicuspid aortic valves. Ann Thorac Surg 2011; 92: $1384-1389$

5. Vergara C, Viscardi F, Antiga L, Luciani GB. Influence of bicuspid valve geometry on ascending aortic fluid dynamics: A parametric study. Artif Organs 2012; 36: 368-378.

6. Balistreri CR, Pisano C, Candore G, Maresi E, Codispoti M, Ruvolo G. Focus on the unique mechanisms involved in thoracic aortic aneurysm formation in bicuspid aortic valve versus tricuspid aortic valve patients: Clinical implications of a pilot study. Eur J Cardiothorac Surg 2013; 43: e180-e186.

7. Girdauskas E, Rouman M, Borger MA, Kuntze T. Comparison of aortic media changes in patients with bicuspid aortic valve stenosis versus bicuspid valve insufficiency and proximal aortic aneurysm. Interact Cardiovasc Thorac Surg 2013; 17: 931-936.

8. Nataatmadja M, West M, West J, Summers K, Walker P, Nagata M, et al. Abnormal extracellular matrix protein transport associated with increased apoptosis of vascular smooth muscle cells in marfan syndrome and bicuspid aortic valve thoracic aortic aneurysm. Circulation 2003; 108(Suppl 1): II329II334.

9. Wagsater D, Paloschi V, Hanemaaijer R, Hultenby K, Bank RA, Franco-Cereceda A, et al. Impaired collagen biosynthesis and cross-linking in aorta of patients with bicuspid aortic valve. $\mathrm{J} \mathrm{Am}$ Heart Assoc 2013; 2: e000034.

10. Biner S, Rafique AM, Ray I, Cuk O, Siegel RJ, Tolstrup K Aortopathy is prevalent in relatives of bicuspid aortic valve patients. J Am Coll Cardiol 2009; 53: 2288-2295.

11. Pasta S, Phillippi JA, Gleason TG, Vorp DA. Effect of aneurysm on the mechanical dissection properties of the human ascending thoracic aorta. J Thorac Cardiovasc Surg 2012; 143: 460-467.

12. Borger MA, Preston M, Ivanov J, Fedak PW, Davierwala P, Armstrong S, et al. Should the ascending aorta be replaced more frequently in patients with bicuspid aortic valve disease? J Thorac Cardiovasc Surg 2004; 128: 677-683.

13. Garg V, Muth AN, Ransom JF, Schluterman MK, Barnes R, King IN, et al. Mutations in NOTCH1 cause aortic valve disease. Nature 2005; 437: 270-274.

14. Koenig SN, Bosse KM, Nadorlik HA, Lilly B, Garg V. Evidence of aortopathy in mice with haploinsufficiency of Notch1 in Nos3-null background. J Cardiovasc Dev Dis 2015; 2: 17-30.

15. Padang R, Bagnall RD, Richmond DR, Bannon PG, Semsarian C. Rare non-synonymous variations in the transcriptional activation domains of GATA5 in bicuspid aortic valve disease. $J$ Mol Cell Cardiol 2012; 53: 277-281.

16. Foffa I, Ait Ali L, Panesi P, Mariani M, Festa P, Botto N, et al. Sequencing of NOTCH1, GATA5, TGFBR1 and TGFBR2 genes in familial cases of bicuspid aortic valve. BMC Med Genet 2013; 14: 44

17. Qu XK, Qiu XB, Yuan F, Wang J, Zhao CM, Liu XY, et al. A novel NKX2.5 loss-of-function mutation associated with congenital bicuspid aortic valve. Am J Cardiol 2014; 114: 1891-1895.

18. Huang da W, Sherman BT, Lempicki RA. Systematic and integrative analysis of large gene lists using DAVID bioinformatics resources. Nat Protoc 2009; 4: 44-57.

19. Jiao J, Xiong W, Wang L, Yang J, Qiu P, Hirai H, et al. Differentiation defect in neural crest-derived smooth muscle cells in patients with aortopathy associated with bicuspid aortic valves. EBioMedicine 2016; 10: 282-290.

20. Li W, Chen Q, Mills I, Sumpio BE. Involvement of S6 kinase and p38 mitogen activated protein kinase pathways in strain-induced alignment and proliferation of bovine aortic smooth muscle cells. $J$ Cell Physiol 2003; 195: 202-209.

21. Jiao Y, Li G, Li Q, Ali R, Qin L, Li W, et al. mTOR (Mechanistic Target of Rapamycin) inhibition decreases mechanosignaling, collagen accumulation, and stiffening of the thoracic aorta in elastin-deficient mice. Arterioscler Thromb Vasc Biol 2017; 37: $1657-1666$

22. Antoine M, Wirz W, Tag CG, Mavituna M, Emans N, Korff T, et al. Expression pattern of fibroblast growth factors (FGFs), their receptors and antagonists in primary endothelial cells and vascular smooth muscle cells. Growth Factors 2005; 23: 87-95.

23. Joannes A, Brayer S, Besnard V, Marchal-Somme J, Jaillet M, Mordant P, et al. FGF9 and FGF18 in idiopathic pulmonary fibrosis promote survival and migration and inhibit myofibroblast differentiation of human lung fibroblasts in vitro. Am J Physiol Lung Cell Mol Physiol 2016; 310: L615-L629.

24. Grewal N, Gittenberger-de Groot AC, DeRuiter MC, Klautz RJ, Poelmann RE, Duim S, et al. Bicuspid aortic valve: Phosphorylation of c-Kit and downstream targets are prognostic for future aortopathy. Eur J Cardiothorac Surg 2014; 46: 831839.

25. Liu ZJ, Xiao M, Balint K, Soma A, Pinnix CC, Capobianco AJ, et al. Inhibition of endothelial cell proliferation by Notch1 signaling is mediated by repressing MAPK and PI3K/Akt pathways and requires MAML1. FASEB J 2006; 20: 1009-1011.

26. Brownstein AJ, Ziganshin BA, Kuivaniemi H, Body SC, Bale AE, Elefteriades JA. Genes associated with thoracic aortic aneurysm and dissection: An update and clinical implications. Aorta (Stamford) 2017; 5: 11-20.

27. Arcucci A, Ruocco MR, Albano F, Granato G, Romano V, Corso G, et al. Analysis of extracellular superoxide dismutase and Akt in ascending aortic aneurysm with tricuspid or bicuspid aortic valve. Eur J Histochem 2014; 58: 2383. 